\section{Themenkomplex II: Diagnostik}

S. Müller-Lissner, C. Fibbe, T. Frieling, M. Jung

\section{Symptomatik}

Folgende Beschwerden können durch gastroösophagealen Reflux verursacht werden: „untere“ Refluxsymptome: Sodbrennen (brennendes Gefühl hinter dem Brustbein = heartburn), saures Aufstoßen, nicht saures Aufstoßen (z. B. bei Säuresuppression), retrosternale Schmerzen, Dysphagie (Schluckerschwernis); selten Odynophagie (Schmerz beim Schlucken); „obere“ Refluxsymptome: Brennen im Rachen, saures und nicht saures Aufstoßen (= Regurgitation, oft fälschlich als Erbrechen bezeichnet!), Reizhusten/morgendliches Räuspern, belegte Stimme, Heiserkeit, Reizhusten, Asthmaanfälle. Die Beschwerden werden oft ausgelöst oder verstärkt durch: Essen, längeren Nüchternzustand, süße Speisen, Alkohol, gebückte Körperhaltung, Liegen. Der Verlauf kann episodisch, kontinuierlich oder intermittierend sein. Spontanremissionen sind häufig, ein komplettes dauerhaftes Verschwinden ist aber selten [1,2].

\section{Diagnostischer Wert von Sodbrennen Konsens}

Sodbrennen ist das sensitivste Symptom der gastroösophagealen Refluxerkrankung. Wird Sodbrennen als das führende klinische Symptom angegeben, liegt mit hoher Wahrscheinlichkeit (> 75\%) eine Refluxerkrankung vor. Das Fehlen endoskopischer Veränderungen schließt dagegen eine Refluxerkrankung nicht aus (B).

\section{Kommentar}

Ist Sodbrennen das klinische Hauptsymptom, so besteht meist ein pathologisch gesteigerter Reflux [1,3]. Wird dagegen neben anderen Symptomen auch Sodbrennen angegeben, so sind andere Erkrankungen wie funktionelle Dyspepsie oder Ulkus ähnlich wahrscheinlich [4]. Der fehlende Goldstandard für die Diagnose der Refluxerkrankung bei unauffälliger Endoskopie erschwert die Einschätzung des prädiktiven Wertes der klinischen Symptomatik in der Diagnosestellung der Refluxerkrankung [5].

\section{Diagnostischer Wert anderer Symptome Konsens}

Unspezifische Symptome wie Dysphagie, retrosternale Schmerzen und respiratorische Symptome können das klinische Beschwerdebild der Refluxerkrankung dominieren und eine rein anamnestische Diagnosestellung erschweren (B).

\section{Kommentar}

Motilitätsstörungen des tubulären Ösophagus und peptische Strikturen sind Komplikationen einer langjährigen Refluxerkrankung und können Dysphagie und retrosternale Schmerzen in den Vordergrund der klinischen Symptomatik rücken. Daneben können auch extraösophageale Manifestationen der Refluxerkrankung, insbesondere pulmonale Erkrankungen, das Beschwerdebild dominieren. Die Abgrenzung zu anderen Krankheitsgruppen kann in diesen Fällen schwierig sein und eine weitere Diagnostik erfordern [6-8].

\section{Beschwerdenmuster}

\section{Konsens}

Weder Art, Intensität und Häufigkeit von Refluxbeschwerden noch deren zeitliches Auftreten (nüchtern, nach der Nahrungsaufnahme, nachts) erlaubt einen Rückschluss auf den Schwere- 
grad der gastroösophagealen Refluxerkrankung (z.B. Vorliegen und Ausmaß einer Ösophagitis) (B).

\section{Kommentar}

Untersuchungen zur zeitlichen Korrelation von klinischen Symptomen mit einem gastroösophagealen Reflux in der 24-h-pH-Metrie konnten zeigen, dass Refluxbeschwerden wie auch die meisten Refluxepisoden vor allem nach dem Essen auftreten, seltener nachts [9]. Während Patienten mit einer Refluxösophagitis zwar eine höhere kumulative und nächtliche Refluxdauer, eine vermehrte Anzahl langer Refluxe und eine längere Refluxdauer aufweisen [10], lässt umgekehrt ein Vorhandensein dieser Phänomene keinen Rückschluss auf das Ausmaß der ösophagealen Schädigung zu [11 - 13].

\section{Endoskopie}

\section{Indikation}

Konsens

Auch bei eindeutiger Refluxsymptomatik und fehlenden Alarmsymptomen ist eine frühe Endoskopie zu empfehlen, desgleichen wenn eine Refluxerkrankung differenzialdiagnostisch in Erwägung gezogen wird (B).

\section{Kommentar}

Die Endoskopie ermöglicht die primäre Diagnose der Refluxösophagitis und die Festlegung ihres Schweregrades [14]. Sie erleichtert damit die Therapieplanung und eine exakte Therapiekontrolle. Als Indexendoskopie ist sie von Vorteil für die Diagnostik des Barrett-Ösophagus und die Erfassung von Komplikationen (Ulkus, Striktur). Gleichzeitig können Erkrankungen, die von einer Frühtherapie profitieren, ausgeschlossen werden, in erster Linie Malignome. Die Endoskopie kann zusätzlich zu Patientenzufriedenheit und Wirtschaftlichkeit in der Behandlung von Patienten mit Refluxkrankheit führen [15].

\section{Erosionen}

Konsens

Fleckige, streifige oder zirkulär konfluierende Epitheldefekte (Erosionen) im distalen Ösophagus oder im Anschluss an die Z-Linie erlauben die Diagnose einer Refluxösophagitis (ERD) (B).

\section{Kommentar}

Fleckige, streifige oder zirkulär konfluierende Epitheldefekte (Erosionen) im distalen Ösophagus oder im Anschluss an die Z-Linie sind allgemein akzeptierte makroskopische Zeichen einer Refluxösophagitis [14]. Sie sind praktisch immer von einer pathologischen pH-Metrie begleitet [16]. Andere makroskopische Befunde sowie histologische Veränderungen sind in ihrem Wert umstritten.

\section{Klassifikationssysteme}

\section{Konsens}

Zur endoskopischen Einteilung der Refluxösophagitis für klinische Belange existieren drei gängige Klassifikationen: die Klassifikation nach Savary-Miller, die Los-Angeles- und die MUSEKlassifikation. Prinzipiell sind alle Klassifikationen akzeptabel. Im Befund ist die verwendete Klassifikation anzugeben (B).

\section{Kommentar}

Die „traditionelle“ Savary-Miller-Klassifikation orientiert sich ebenso wie die Los-Angeles-Klassifikation an dem Ausmaß der Mukosaläsionen in der distalen Speiseröhre [14, 17]. Die SavaryMiller-Klassifikation unterscheidet drei, die Los-Angeles-Klassifikation vier Schweregrade. Dabei korreliert Grad I der Savary-Miller-Klassifikation mit Grad A und B der Los-Angeles-Klassifikation, die sich nur in der Größe der Mukosaläsion ( $<5 \mathrm{~mm} />5 \mathrm{~mm}$ ) unterscheidet. Grad II nach Savary-Miller entspricht weitest gehend Grad C der Los-Angeles-Klassifizierung (konfluierende Mukosaläsionen bis zu 75\% des Gesamtumfangs), Grad III entspricht Grad D (Mukosaläsionen mit mindestens 75\% des Gesamtumfangs der Speiseröhre). Die MUSE-Klassifikation (Metaplasie - Ulzeration Striktur - Erosion) definiert klar die endoskopisch diagnostizierten Läsionen und gibt die detaillierteste Beschreibung der Refluxveränderungen in der Speiseröhre [18].

Während die MUSE-Klassifikation sich durch die Detailliertheit am besten für den Vergleich von Befunden bei aufeinander folgenden Endoskopien eignet, erscheint die Los-Angeles-Klassifikation durch ihre einfache Beschreibung am praktikabelsten. Sie wird auch international häufig eingesetzt.

\section{Weitere Aussagekraft der Endoskopie Konsens}

Über den qualitativen Nachweis von Erosionen hinaus ist die Endoskopie bei der Diagnostik der Refluxkrankheit besonders wertvoll im Grading der Ösophagitis und im Grading der Veränderungen unter Therapie. Die Beschreibung einer Hiatushernie spielt für die Diagnosestellung keine Rolle. Sie ist aber für die Operationsplanung wichtig (Herniengröße). Einer klaffenden Kardia oder einem unvollständigen Schluss des gastroösophagealen Übergangs bzw. einer insuffizienten Umschließung des Endoskops kommt keine diagnostische Wertigkeit zu (B).

\section{Kommentar}

Zwar besteht eine Beziehung zwischen Herniengröße und dem Schweregrad einer evtl. bestehenden Ösophagitis [19], dies bringt aber keinen diagnostischen Zugewinn. Die Beschreibung einer klaffenden Kardia, des unvollständigen Schlusses des gastroösophagealen Übergangs bzw. einer insuffizienten Umschließung des Endoskops ist subjektiv. Einzelne Autoren beschreiben ein makroskopisches Grading des gastroösophagealen Verschlussmechanismus (Valve), wobei die Schwere von endoskopisch sichtbaren Schleimhautläsionen (Refluxösophagitis) mit der Insuffizienz des Verschlusses („klaffende Kardia“) korrelieren soll [20]. Diese Beobachtung blieb aber bisher unbestätigt.

\section{Zusätzliche Diagnostik bei ERD \\ Konsens}

Beim endoskopischen Nachweis einer Refluxösophagitis ist zunächst keine weitere Diagnostik angezeigt (B).

\section{Kommentar}

Die makroskopischen Läsionen sind derart spezifisch, dass mit ihrem Nachweis die Diagnose „Refluxösophagitis“ ausreichend belegt ist [14]. Eine zusätzliche diagnostische Maßnahme optimiert nicht die diagnostische Sicherheit. Aus den Befunden der pH-Metrie und Ösophagusmanometrie lassen sich zunächst keine differenzialtherapeutischen Konsequenzen ableiten. Bei 
symptomatischer Refluxösophagitis wird daher ohne weitere Funktionsdiagnostik medikamentös therapiert.

\section{Minimalbefunde \\ Konsens}

Bei Abwesenheit von Erosionen erlauben Minimalbefunde wie Erythem, Granulation, undeutlicher Übergang des Schleimhautbereichs von Plattenepithel zu Zylinderepithel, verstärkte Gefäßzeichnung im distalen Ösophagus, Ödem oder Hervorhebung der mukosalen Falten für sich allein nicht die Diagnose „Refluxkrankheit“"(B).

\section{Kommentar}

Die genannten minimalen Veränderungen sind nicht spezifisch für sauren Reflux [21-23]. Lediglich für rote Streifen (red streaks) scheint eine Korrelation zu Refluxsymptomen zu bestehen. Sie geben histologisch regenerative Veränderungen des Plattenepithels wieder und werden als histologisches Korrelat zur peptischen Schädigung der Mukosa gewertet [24]. Weiterhin fand sich für diese Veränderungen in einer Interobserver-Studie eine schlechte Übereinstimmung mit katastrophal niederen Kappawerten (Kappa 0,00-0,09) [23].

\section{Bioptische Diagnostik bei NERD Konsens}

Eine endoskopische Biopsie spielt für die Diagnose der NERD keine Rolle (B).

\section{Kommentar}

In frühen Studien wurden histologische Veränderungen des nicht erodierten Epithels bei Refluxösophagitis beschrieben wie Verlängerung der Papillen, Verdickung der Basalzellschicht und entzündliche Zellinfiltration [25]. Das Material war mittels Saugbiopsie gewonnen worden. Diese Ergebnisse konnten in einer anderen Studie nicht bestätigt werden [26]. Kontrollierte Serien mittels konventioneller Zangenbiopsien und unter Verblindung des befundenden Pathologen ergaben, dass die Histologie nicht in der Lage war, Patienten mit gesicherter NERD von Nichtrefluxpatienten zu unterscheiden [16, 27]. Die Biopsie wird deshalb zur Diagnostik von GERD als nicht geeignet betrachtet [14].

\section{Biopsie bei bekannter Refluxkrankheit Konsens}

Bei Erosion, Erythem, Schatzki-Ring und ringförmig verdicktem Ösophagus wird keine Biopsie empfohlen, soweit ein BarrettÖsophagus ausgeschlossen werden kann (B).

\section{Kommentar}

Die bioptische Diagnostik bei Barrett-Ösophagus wird an anderer Stelle abgehandelt. Unstrittig ist die Biopsie bei Ulzera und exophytischen Läsionen, auch bei Stenose wird sie zum Malignomausschluss empfohlen. Bei den anderen genannten Läsionen (Erosion, Erythem, Schatzki-Ring und ringförmig verdicktem Ösophagus) kann von einer Biopsie kein diagnostischer Gewinn erwartet werden [28].

\section{Probetherapie}

\section{Begriff}

Im Zusammenhang mit oberen gastrointestinalen Symptomen und Refluxkrankheit sind drei verschiedene Begriffe von Probetherapie in Gebrauch, die nicht verwechselt werden dürfen:

1. Probetherapie bei undiagnostizierter Dyspepsie zur Verhinderung „unnötiger“ Endoskopien. Die zugrunde liegende Hypothese lautet: Bei erfolgreicher Behandlung entfällt die invasive und teure Diagnostik. Diese Art der Probetherapie hat sich nicht als effektiv erwiesen [29, 30]. Sie steht hier im Übrigen nicht zur Diskussion.

2. Probetherapie bei Symptomen, die bei negativer Endoskopie verdächtig auf Refluxkrankheit sind. Die zugrunde liegende Hypothese lautet: In dieser Situation sprechen nur Refluxbeschwerden auf eine säuresuppressive Therapie an (Ex-juvantibus-Diagnose der Refluxkrankheit).

3. Probetherapie zur Klärung der Ursächlichkeit von Reflux für respiratorische Symptome. Beide Diagnosen stehen schon fest (Refluxkrankheit und Asthma bzw. chronischer Husten). Die zugrunde liegende Hypothese lautet: Wenn sich unter Refluxtherapie die respiratorischen Symptome bessern, besteht ein kausaler Zusammenhang. Dazu siehe bei „extraösophagale Manifestationen“.

\section{Indikation}

\section{Konsens}

Die Probetherapie zur Ex-juvantibus-Diagnose der Refluxkrankheit ist indiziert bei Symptomen, die vereinbar mit, aber nicht spezifisch für eine Refluxkrankheit sind (z.B. epigastrische oder retrosternale Schmerzen oder Druck), und unauffälliger Endoskopie (A).

\section{Kommentar}

Bei endoskopisch sichtbarer Refluxösophagitis (ERD) darf deren Ursächlichkeit für die Symptome zunächst angenommen werden. Nur wenn sich die Symptome unter Therapie nicht innerhalb weniger Tage deutlich bessern, sind Zweifel angebracht. Sodbrennen als Leitsymptom ist ausreichend spezifisch für GERD, um auch bei unauffälligem Endoskopiebefund die Diagnose zu gestatten [1]. Daher reduziert sich der Einsatz der Probetherapie zur Ex-juvantibus-Diagnose der Refluxkrankheit auf Symptome, die vereinbar mit, aber nicht spezifisch für eine Refluxkrankheit sind, bei unauffälliger Endoskopie. Studien zu diesem Thema verwendeten unterschiedliche Dosen von PPI, Therapiedauern und Erfolgskriterien. Unter hohen Dosen von PPI erwiesen sich die Sensitivität und (selten ermittelte) Spezifität als $70-90 \%$ [31-37]. Als Goldstandard diente meist die pHMetrie. Bei einem direkten Vergleich von Probetherapie und $\mathrm{pH}-$ Metrie bei Patienten mit Refluxösophagitis waren beide Verfahren ähnlich zuverlässig [38]. Die Probetherapie verlangt keine apparative Investition, ist nicht invasiv, aber wesentlich langwieriger als die $\mathrm{pH}-$ Metrie.

\section{Medikament Konsens}

Bei der Probetherapie zur Ex-juvantibus-Diagnose der Refluxkrankheit sollten ausschließlich Protonenpumpenblocker angewandt werden (A). Die Dosierung der Probetherapie sollte mit 
der zwei- bis dreifachen zur Refluxtherapie empfohlenen Standarddosis erfolgen (B).

\section{Kommentar}

Für die Dauer der Probetherapie wird eine möglichst komplette Säuresuppression angestrebt. Diese ist mit den üblichen Dosierungen der PPIs nicht sicher gewährleistet [39].

\section{Dauer}

\section{Konsens}

Die Dauer der Probetherapie sollte eine bis zwei Wochen betragen (B).

\section{Kommentar}

Aus zahlreichen Studien ist bekannt, dass die Refluxsymptomatik unter einer wirksamen Therapie binnen weniger Tage drastisch abnimmt (z.B. [40-42]). Da die Intensität von Refluxbeschwerden aber auch ohne Therapie von Tag zu Tag variiert, erlaubt eine Betrachtung von nur wenigen Tagen keine zuverlässige Einschätzung des Erfolgs bzw. Misserfolgs einer Probetherapie. Eine Dauer von ein bis zwei Wochen hat sich in den entsprechenden Studien als geeignet erwiesen [31 -37].

\section{Zielkriterium}

Konsens

Die Probetherapie (Ex-juvantibus-Diagnose der Refluxkrankheit) gilt als erfolgreich (d.h. NERD als gesichert), wenn weit gehende Besserung von pharyngealen, retrosternalen und epigastrischen Symptomen erzielt wird (z.B. 75\% Besserung auf einer visuellen Analogskala oder Reduktion auf Grad 1 oder 2 auf einer 5-Punkte-Likert-Skala) (B).

\section{Kommentar}

Komplette Beschwerdefreiheit ist weder in kontrollierten Therapiestudien noch in Studien zur Probetherapie zu erreichen [31-33, 35-37, 40-42]. Zur Objektivierung und Dokumentation erscheint der Versuch einer Quantifizierung mittels der genannten Instrumente empfehlenswert.

\section{pH-Metrie der Speiseröhre}

Die pH-Metrie der Speiseröhre gilt als der Goldstandard für die quantitative Erfassung der Säurebelastung des unteren Ösophagus. Ihr Einsatz setzt eine vorherige obere Endoskopie voraus.

\section{Indikation}

\section{Konsens}

Zur Diagnosesicherung der gastroösophagealen Refluxerkrankung sollte eine pH-Metrie der Speiseröhre bei denjenigen Patienten durchgeführt werden, deren Refluxbeschwerden gegenüber einer Therapie mit PPI in üblicher Dosis refraktär sind (siehe Probetherapie) (B).

\section{Kommentar}

Die diagnostische Sensitivität der 24-h-pH-Metrie wird oft überschätzt. So muss sowohl bei bis zu 25\% der Patienten mit einer Refluxösophagitis als auch bei einem Drittel der Patienten mit nichterosiver Refluxerkrankung mit quantitativ normalen Werten gerechnet werden. Denn die Von-Tag-zu-Tag-Variation von
Reflux ist so groß, dass auch bei eindeutiger Refluxkrankheit Tage mit normalen Refluxwerten vorkommen. Außerdem entgehen Patienten mit hypersensitivem Ösophagus (refluxassoziierte Beschwerden bei quantitativ „normalem“ Reflux) der pHMetrie, wenn kein Symptomenindex erstellt wird (s.u.) [43]. Die 24-h-pH-Metrie kann eingesetzt werden, um bei Persistenz von Refluxbeschwerden unter adäquater (PPI-)Medikation eine ungenügende Säureblockade von einer inkorrekten NERD-Diagnose zu differenzieren [44-46]. Hierbei besteht manchmal eine nur scheinbare Therapieresistenz, denn eine vielfach mit der Refluxerkrankung assoziierte Verzögerung der Magenentleerung kann zu einer ungenügenden Wirkstofffreisetzung und damit zu einer fehlenden Säuresuppression führen. In diesen Fällen kann eine bipolare Säuremessung (Ösophagus und Magen) hilfreich sein (fehlende Säuresuppression im Magen und persistierender Reflux). Die pH-Metrie kann auch bei der Abklärung einer rekurrenten Refluxsymtomatik nach Antirefluxchirurgie sinnvoll sein $[47,48]$. Sie ist dagegen nicht geeignet, eine Refluxösophagitis zu diagnostizieren oder zu bestätigen [49].

\section{Technische Aspekte \\ Konsens}

Glas- und Antimonelektroden sind gleichwertig. Bei Vergleichsuntersuchungen sollte jedoch immer derselbe Elektrodentyp verwendet werden (A). Für klinische Fragestellungen ist eine 1-Kanal-Messung im Ösophagus ausreichend (B).

Die Messelektrode wird transnasal eingeführt und $5 \mathrm{~cm}$ oberhalb des Oberrandes des unteren Ösophagussphinkters (UÖS) platziert. Dazu sollte in erster Linie die Ösophagusmanometrie benutzt werden, alternativ der pH-Umsprung zwischen Magen und Ösophagus (B).

Die pH-Messung sollte 24 Stunden dauern (B). Sie kann ambulant oder stationär erfolgen (A).

Zur Beurteilung des gastroösophagealen Refluxes sollte die relative Refluxdauer (Zeit-pH $<4$ in \% der Messzeit) getrennt nach aufrechter und liegender Position herangezogen werden (B).

\section{Kommentar}

Der Vorteil der Antimonelektroden liegt in der geringeren Patientenbelästigung durch den kleineren Durchmesser und die flexiblere Sondenspitze sowie in den geringeren Kosten. Da die Reproduzierbarkeit der Daten für die Glaselektrode höher liegt, sollten diese für wissenschaftliche Zwecke bevorzugt werden [50-52].

Die Verwendung proximaler Messpunkte in der Evaluierung von Patienten mit oberen respiratorischen Symptomen erscheint von limitiertem Wert, da keine $\mathrm{pH}$-Unterschiede zwischen Patienten mit und ohne laryngeale Symptome gefunden wurden [53, 54].

Abweichungen des Abstands von der Kardia können erhebliche Veränderungen der Messwerte hervorrufen. Die Ösophagusmanometrie ist die genaueste Methode zur Lokalisation des Oberrandes des UÖS und sollte zusammen mit dem Nachweis des pH-Umsprungs zwischen Magen und Ösophagus (Vermeidung eines Umschlagens der Sonde) zur Sondenplatzierung benutzt werden $[44,55]$. 
Die Bestimmung des pH-Umsprungs ist insbesondere bei großer Hiatushernie und stark vermindertem Druck im UÖS fehlerbehaftet [56]. Antimonelektroden neigen zum Umschlagen und erfordern manchmal eine radiologische Kontrolle.

Die pH-Metrie muss mindestens 12 Stunden dauern und eine Schlaf- bzw. Liegeperiode enthalten $[57,58]$.

Es wurde eine Vielzahl von Zielvariablen für die pH-Metrie vorgeschlagen, z. B. kumulative Refluxdauer $\mathrm{pH}<4$, Refluxdauer unter der Kurve $\mathrm{pH}<4$, DeMeester-Score, Anzahl langer Refluxe ( $>5$ min, $<$ pH 4), Refluxdauer im Liegen (\%-pH $<4$ ), Refluxdauer aufrecht (\%-pH < 4), Refluxdauer postprandial (\%-pH < 4), längste Refluxepisode (min) [45, 55, 57, 59-61].

Die Erfassung der kumulativen Refluxdauer gesamt und getrennt in aufrechter und liegender Position ist einfach, im Gegensatz zum DeMeester-Score ohne zusätzliche Software möglich und für die Beurteilung des gastroösophagealen Refluxes in der Praxis ausreichend.

\section{Symptomindex}

\section{Konsens}

Die Erstellung eines Symptomindex (Assoziation zwischen Refluxsymptomen und Refluxepisoden) erhöht die Sensitivität der 24-h-pH-Metrie in der Diagnose der Refluxerkrankung (B).

\section{Kommentar}

Insbesondere können refluxbedingte Beschwerden bei quantitativ normalen Refluxwerten identifiziert werden („hypersensitiver Ösophagus“) [62-65]. Eine Einschränkung dieser sinnvollen Methode liegt darin, dass die Erfassung der genauen zeitlichen Korrelation zwischen Beschwerden und Reflux bisweilen nicht möglich ist und die optimale Computersoftware zur Erstellung eines Index nicht überall zur Verfügung steht. Daneben beruhen alle bislang verwendeten Scores auf retrospektiven Analysen und wurden bisher nicht an einem größeren Patientenkollektiv validiert.

\section{Klinische Bedeutung der pH-Metrie Konsens}

Die 24-h-pH-Metrie beeinflusst die Diagnose, Klassifizierung und Behandlung der gastroösophagealen Refluxerkrankung nur bei einem Teil der Patienten signifikant (B).

\section{Kommentar}

Das Ergebnis der Säuremessung ist nach Anamnese und Endoskopie meist vorhersehbar. Die 24-h-ph-Metrie beeinflusst daher die Diagnose und die akute Behandlung der Refluxerkrankung nur bei einigen Patienten $(5-25 \%)$, d.h. bei manchen Patienten mit negativem Endoskopiebefund und atypischen und/oder therapierefraktären Symptomen. Eine auf das Ergebnis der pH-Metrie zurückzuführende Änderung der Medikation führt nur in wenigen Fällen zu einer Verbesserung der Symptomatik und wird häufig nicht über einen langen Zeitraum beibehalten [66].
Manometrie

\section{Aussagekraft \\ Konsens}

Die Ösophagusmanometrie kann die Kompetenz des unteren Ösophagussphinkters (Ruhedruck, Länge) zwischen Magen und Ösophagus und die tubuläre Motilität der Speiseröhre sicher erfassen. Hierbei sollte der Druck im unteren Ösophagussphinkter mittels Sleeve-Kathetern oder radiären Druckaufnehmern gemessen werden. Die Ösophagusmanometrie kann mittels preiswerter „low compliance“-Wasserperfusionssystemen oder teuren elektronischen Druckaufnahmesystemen durchgeführt werden. Für klinische Belange kommt nur die stationäre (= ortsgebundene Kurzzeit-)Manometrie infrage (B).

\section{Kommentar}

Die Messung des unteren Ösophagussphinkter-Ruhedrucks ist von der verwendeten Technik (schneller Durchzug, stationärer Durchzug, Sleeve-Katheter) abhängig. Aus diesem Grund sollte jedes Labor eigene Normwerte etablieren $[67,68]$. Die tubuläre Speiseröhre gilt bei Kontraktionsamplituden kleiner $30 \mathrm{~mm} \mathrm{Hg}$ als hypomotil und ein unterer Ösophagussphinkter-Ruhedruck kleiner $5 \mathrm{~mm} \mathrm{Hg}$ als erniedrigt [67]. Der Vorteil der elektronischen Druckaufnahmesysteme, nämlich ambulante Langzeitmessungen durchführen zu können, spielt bei der gastroösophagealen Refluxerkrankung in der Klinik keine Rolle.

\section{Rolle der Manometrie bei der Refluxkrankheit Konsens}

Die Ösophagusmanometrie spielt bei der primären Diagnose, Klassifizierung und/oder Behandlung der gastroösophagealen Refluxerkrankung keine Rolle (A).

\section{Kommentar}

Obwohl die gastroösophageale Refluxerkrankung durch Motilitätsstörungen verursacht wird, konnte bisher in keiner Untersuchung gezeigt werden, dass eine nachgewiesene Dysmotilität die Diagnose/Klassifizierung der Refluxerkrankung bzw. den therapeutischen Einsatz von Medikamenten signifikant beeinflusst [67]. Die Ösophagusmanometrie spielt auch bei der Indikationsstellung zur Antirefluxoperation keine Rolle, da die präoperativen Messergebnisse des unteren Ösophagussphinkters bzw. der tubulären Ösophagusperistaltik die postoperativen Ergebnisse (Therapie des Refluxes, postoperative Dysphagie) nicht vorhersagen können $[69,70]$. Die Ösophagusmanometrie kann aber im Einzelfall zur differenzialdiagnostischen Abklärung von Dysphagie und Brustschmerz und in diesen Fällen auch zur präoperativen weiterführenden Diagnostik (Lokalisierung des unteren Ösophagussphinkters, Charakterisierung der tubulären Motilität) hilfreich sein [48, $71-75]$.

\section{Röntgenuntersuchung}

\section{Konsens}

Der „Bariumbreischluck“ ist zur Diagnosestellung Refluxkrankheit ungeeignet (B). 


\section{Kommentar}

Bei der radiologischen Refluxdokumentation mittels Bariumbrei wird mittels Provokationsmanövern (Kopftief- und Bauchlage, Valsalva-Pressversuch) versucht, Reflux zu erzeugen. Folgende Argumente sprechen a priori gegen einen diagnostischen Wert des Verfahrens: 1. Da Reflux physiologisch ist, lässt sich aus der rein qualitativen Refluxdarstellung kein Krankheitswert ableiten. 2. Das intermittierende Auftreten von Reflux und seine Abhängigkeit von der Magenfüllung und der Körperlage verlangen eine langzeitige Refluxmessung und keine Momentaufnahme. 3. Die verwendeten Provokationsmanöver sind realitätsfern. Der direkte Vergleich von Bariumbreischluck und pH-Metrie bestätigte die diagnostische Wertlosigkeit der Röntgenuntersuchung für den Nachweis von pathologischem Reflux [76].

\section{Konsens}

Der „Bariumbreischluck“ ist präoperativ vor Fundoplicatio und zur Abklärung postoperativer Syndrome zu empfehlen (B/C).

\section{Kommentar}

Der „Bariumbreischluck“ ist der Goldstandard zum Nachweis der axialen Hiatushernie. Die Diagnosestellung der Refluxkrankheit hängt zwar nicht vom Herniennachweis ab [76, 77], jedoch wird es von manchen Operateuren als hilfreich empfunden, wenn die Anatomie des gastroösophagealen Übergangs bei der Fundoplicatio bekannt ist.

Postoperative Syndrome nach Fundoplicatio sind oft schwierig zu klären und zu behandeln. Für eine befriedigende Einordnung sind meist morphologische und funktionsanalytische Verfahren erforderlich (Endoskopie, Bariumbreischluck, pH-Metrie, Manometrie) [78]. Mit dem Bariumbreischluck lässt sich die Manschette besser beurteilen als endoskopisch (Auflösung, Teleskop).

\section{Duodenogastroösophagealer Reflux}

\section{Begriff}

\section{Konsens}

Die Begriffe „alkalischer Reflux“ und „biliärer Reflux“ sind Fehlbegriffe und sollten durch „duodenogastroösophagealer Reflux“ ersetzt werden (B).

\section{Kommentar}

Der duodenogastroösophageale Reflux ist durch Rückfluss von Dünndarminhalt in den Magen und in die Speiseröhre definiert. Da der Dünndarminhalt immer mit Mageninhalt vermischt wird, kann der Rückfluss vom Dünndarm grundsätzlich alle pH-Werte erreichen und korreliert nicht mit alkalischen $\mathrm{pH}$-Werten. Der Begriff „alkalischer Reflux“ sollte daher vermieden werden. Ebenso ist die Galle nur ein Bestandteil des Dünndarminhaltes, weswegen auch der Begriff „biliärer Reflux“ vermieden werden sollte [79-81].

\section{Quantifizierung \\ Konsens}

Es gibt derzeit keine verlässliche Methode zur Quantifizierung des duodenogastroösophagealen Refluxes. In der Klinik ist we- der die 24-h-pH-Metrie noch die Bilimetrie zur Erkennung eines duodeno-gastroösophagealen Refluxes indiziert (B).

\section{Kommentar}

Bilirubin und seine Konzentration können zuverlässig mit der Bilimetrie erfasst werden [82-86]. Hierdurch kann aber nicht direkt auf einen duodenalen Reflux schädigender Substanzen geschlossen werden, da der Dünndarminhalt neben Bilirubin toxische Bestandteile wie Gallensalze, Phospholipide und Trypsin enthält. Aufgrund der fehlenden Möglichkeit, einen duodenogastroösophagealen Reflux direkt nachzuweisen, und der fehlenden klinischen Konsequenzen sind in der Klinik weder die pH-Metrie noch die Bilimetrie bei V.a. duodenogastroösophagealem Reflux indiziert [87-92].

\section{Impedanzmessung \\ Konsens}

Die Impedanzmessung spielt in der Klinik zur Abklärung einer gastroösophagealen Refluxerkrankung keine Rolle, da bei Erwachsenen der gastroösophageale Reflux überwiegend sauer ist und mit der pH-Metrie ausreichend erfasst werden kann. Die Impedanzmessung kann bei speziellen klinischen Fragestellungen (Bolustransport, nichtsaurer Reflux, Reflux bei Kindern) hilfreich sein (B).

\section{Kommentar}

Mit der elektrischen Impedanzmessung können Flüssigkeitsbewegungen im Ösophagus unabhängig von deren chemischer Zusammensetzung, also z.B. unabhängig vom pH-Wert, valide gemessen werden [93-98]. Vergleichende Untersuchungen, die einen Vorteil der Impedanzmessung gegenüber pH-Metrie, Bilimetrie, Manometrie und röntgenologischen bildgebenden Verfahren (z.B. Brotschluck, Szintigraphie) aufzeigen, liegen allerdings bisher nicht vor [99-101].

\section{Literatur}

${ }^{1}$ Klauser AG, Schindlbeck NE, Müller-Lissner SA. Symptoms in gastrooesophageal reflux disease. Lancet 1990; 335: 205-208

${ }^{2}$ Schindlbeck NE, Klauser AG, Berghammer G et al. Three year followup of patients with gastro-oesophageal reflux disease. Gut 1992; 33: $1016-1019$

${ }^{3}$ Martinez-Serna T, Tercero FJ, Filipi CJ et al. Symptom priority ranking in the care of gastroesophageal reflux: a review of 1,850 cases. Dig Dis 1999; 17: 219-224

${ }^{4}$ Berstad A, Hatlebakk JG. The predictive value of symptoms in gastrooesophageal reflux disease. Scand J Gastroenterol Suppl 1995; 211 : $1-4$

${ }^{5}$ Johnsson F, Joelsson B, Gudmundsson K et al. Symptoms and endoscopic findings in the diagnosis of gastroesophageal reflux disease. Scand J Gastroenterol 1987; 22: 714-718

${ }^{6}$ Garcia-Compean D, Gonzalez MV et al. Prevalence of gastroesophageal reflux disease with extraesophageal symptoms referred from otolaryngology, allergy, and cardiology practices: a prospective study. Dig Dis 2000; 18: $178-182$

${ }^{7}$ Guedon C. How to diagnose gastroesophageal reflux? Part 1 Diagnostic value of symptoms. Interpretation and role of endoscopy. Gastroenterol Clin Biol 1999; 23: S202-S207

${ }^{8}$ Muller-Lissner. Diagnostic value of symptoms in gastroesophageal reflux disease. Gastroenterol Clin Biol 1999; 23: S13-S16

${ }^{9}$ Gudmundsson K, Johnsson F, Joelsson B. The time pattern of gastroesophageal reflux. Scand J Gastroenterol 1988; 23: 75 - 79

${ }^{10}$ Robertson DA, Aldersley MA et al. Patterns of acid reflux in complicated oesophagitis. Gut 1987; 28: $1484-1488$ 
${ }^{11}$ Galmiche JP, Barthelemy P et al. Treating the symptoms of gastrooesophageal reflux disease: a double-blind comparison of omeprazole and cisapride. Aliment Pharmacol Ther 1997; 11: 765 - 773

${ }^{12}$ Lind T, Havelund T et al. Heartburn without oesophagitis: efficacy of omeprazole therapy and features determining therapeutic response. Scand J Gastroenterol 1997; 32: $974-979$

${ }^{13}$ Smout AJPM. Endoscopic-negative acid reflux disease. Aliment Pharmacol Ther 1997; 11 (suppl): 81 - 85

${ }^{14}$ Dent J, Brun J, Fendrick A et al. An evidence-based appraisal of reflux disease management - the Genval Workshop Report. Gut 1999; 44: $\mathrm{S} 1-\mathrm{S} 16$

${ }^{15}$ Hotz J, Madisch A, Classen M et al. Internationaler Konsens zur Refluxkrankheit der Speiseröhre. Dtsch Med Wschr 2000; 125: 1308-1312

${ }^{16}$ Schindlbeck NE, Wiebecke B, Klauser AG et al. Diagnostic value of histology in non-erosive gastro-oesophageal reflux disease. Gut 1996; 39: $151-154$

17 Ollyo JB, Gontollier C, Brossard E et al. La nouvelle classification de Savary des oesophagites de reflux. Acta Endoscopica 1992; 22: 307

${ }^{18}$ Armstrong D. Endoscopic evaluation of gastro-esophageal reflux disease. Yale J biol Med 1999; 72: $93-100$

${ }^{19}$ Jones MP, Sloan SS, Rabine JC et al. Hiatal hernia size is the dominant determinant of esophagitis presence and severity in gastroesophageal reflux disease. Am J Gastroenterol 2001; 96 (6): 1711 - 1717

20 Öberg S, Peters JH, DeMeester TR et al. Endoscopic grading of the gastroesophageal valve in patients with symptoms of gastroesophageal reflux disease (GERD). Surg Endosc 1999; 13: 1184-1188

${ }^{21}$ Achem SR. Endoscopy-negative gastroesophageal reflux disease. The hypersensitive esophagus. Gastroenterol Clin North America 1999; 28: $893-904$

22 Galmiche JP, des Varannes SB. Endoscopy-negative reflux disease. Curr Gastroenterol Rep 2001; 3: 206-214

${ }^{23}$ Lundell LR, Dent J, Bennett JR et al. Endoscopic assessment of oesophagitis: clinical and functional correlates and further validation of the Los Angeles classification. Gut 1999; 45: $172-180$

${ }^{24}$ Vieth M, Stolte M. Can an endoscopically negative reflux disease be histologically diagnosed? Z Gastroenterol 2000; 38: 817-818

25 Ismail-Beigi F, Horton PF, Pope CE. Histological consequences of gastroesophageal reflux in man. Gastroenterology 1970; 58: $163-174$

${ }^{26}$ Seefeld U, Krejs GJ, Siebenmann RE et al. Esophageal histology in gastroesophageal reflux: Morphometric findings in suction biopsies. Dig Dis Sci 1977; 22: 956 - 964

${ }^{27}$ Collins JSA, Watt PCH, Hamilton PW et al. Assessment of oesophagitis by histology and morphometry. Histopathology 1989; 14: 381 - 389

${ }^{28}$ Morrow JB, Vargow JJ, Goldblum JR et al. The Ringed Esophagus: Histological Features of GERD. Am J Gastroenterol 2001; 96: 984-989

${ }^{29}$ Bytzer P, Moller Hansen J, de Schaffalitzky Muckadell OB. Empirical H2-blocker therapy or prompt endoscopy in management of dyspepsia. Lancet 1994; 343: 811 - 816

${ }^{30}$ Silverstein MD, Petterson T, Talley NJ. Initial endoscopy or empirical therapy with or without testing for Helicobacter pylori for dyspepsia: a decision analysis. Gastroenterology 1996; 110: $72-83$

${ }^{31}$ Fass R, Fennerty MB, Ofman JJ et al. The clinical and economic value of a short course of omeprazole in patients with noncardiac chest pain. Gastroenterology 1998; 115: $42-49$

32 Jaup BH. Effective acid inhibition as a diagnostic tool for gastroesopheagel reflux disease. Gut 1995; 37 (Suppl 2): A36

33 Johnsson F, Weyvadt L, Solhaug JH et al. One week omeprazole treatment as a diagnostic test for gastro-oesophageal reflux disease. Gut 1996; 39 (Suppl 1): A39

${ }^{34}$ Numans ME, Lau J, de Wit NJ et al. Short-term treatment with protonpump inhibitors as a test for gastroesophageal reflux disease: a metaanalysis of diagnostic test characteristics. Ann Intern Med 2004; 140 : $518-527$

${ }^{35}$ Schenk BE, Kuipers EJ, Klinkenberg-Knol EC et al. Omeprazole as a diagnsotiv tool in gastroesophageal reflux disease. Am J Gastroenterol 1997; 92: 1997-2000

${ }^{36}$ Schindlbeck NE, Klauser AG, Voderholzer WA et al. Empiric therapy for gastroesophageal reflux disease. Arch Intern Med 1995; 155: $1808-12$

37 Zierer MD, Sanowski RA, Young MF et al. Can a single dose of omeprazole be used to identify gastroesophageal reflux and acid-related chest pain? Am J Gastroenterology 1993; 88: 1501
${ }^{38}$ Fass R, Ofman JJ, Sampliner RE et al. The omeprazole test is as sensitive as 24-h oesophageal $\mathrm{pH}$ monitoring in diagnosing gastro-oesophageal reflux disease in symptomatic patients with erosive oesophagitis. Aliment Pharmacol Ther 2000; 14: 389-396

${ }^{39}$ Leite LP, Johnston BT, Just RJ et al. Persistent acid secretion during omeprazole therapy: a study of gastric acid profiles in patients demonstating failure of omeprazole therapy. Am J Gastroenterol 1996; 91 (8): $1527-1531$

40 Maton PN, Orlando R, Joelsson B. Efficacy of omeprazole versus ranitidine for symptomatic treatment of poorly responsive acid reflux disease - a prospective controlled trial. Aliment Pharmacol Ther 1999; 13: $819-826$

${ }^{41}$ Richter JE, Kahrilas PJ, Johanson J et al. Efficacy and safety of esomeprazole compared with omeprazole in GERD patients with erosive esophagitis: a randomized controlled trial. Am J Gastroenterol 2001; 96: $656-665$

${ }^{42}$ Festen HPM, Schenk E, Tan G et al. Omeprazole versus high-dose ranitidine in mild gastroesophageal reflux disease: short- and long-term treatment. Am J Gastroenterol 1999; 94: 931 - 936

${ }^{43}$ Watson RGP, Tham TCK, Johnston BT et al. Double blind cross-over placebo controlled study of omeprazole in the treatment of patients with reflux symptoms and physiological levels of acid reflux - the „sensitive oesophagus“. Gut 1997; 40: 587-590

${ }^{44}$ Ergun GA, Kahrilas PJ. Clinical applications of esophageal manometry and $\mathrm{pH}$ monitoring. Am J Gastroenterol 1996; 91: 1077-1089

${ }^{45}$ Locke GR, Talley NJ. 24-hour monitoring for gastro-oesophageal reflux disease. Lancet 1993; 342: 1246-1247

${ }^{46}$ Richter JE. Typical and atypical presentations of gastroesophageal reflux disease. The role of esophageal testing in diagnosis and management. Gastroenterol Clin North Am 1996; 25: 75 - 102

${ }^{47}$ Fuchs KH, DeMeester TR, Albertucci M. Specificity and sensitivity of objective diagnosis of gastroesophageal reflux disease. Surgery 1987; 102: $575-580$

48 Waring JP, Hunter JG, Oddsdottir M et al. The preoperative evaluation of patients considered for laparoscopic antireflux surgery. Am J Gastroenterol 1995; 90: 35 - 38

${ }^{49}$ Ghillebert G, Demeyere AM, Janssens J et al. How well can quantitative 24-hour intraesophageal $\mathrm{pH}$ monitoring distinguish various degrees of reflux disease? Dig Dis Sci 1995; 40: 1317-1324

50 Ask P, Edwall G, Johansson KE. Accuracy and choice of procedures in 24-hour oesophageal $\mathrm{pH}$ monitoring with monocrystalline antimony electrodes. Med Biol Eng Comput 1986; 24: 602 - 608

${ }^{51}$ Richter JE, Bradley LA, DeMeester TR et al. Normal 24-hr ambulatory esophageal $\mathrm{pH}$ values. Influence of study center, $\mathrm{pH}$ electrode, age, and gender. Dig Dis Sci 1992; 37: 849-856

52 Vandenplas Y, Helven R, Goyvaerts H. Comparative study of glass and antimony electrodes for continuous oesophageal $\mathrm{pH}$ monitoring. Gut 1991; 32: 708-712

53 Dobhan R, Castell DO. Normal and abnormal esophageal acid exposure: results of ambulatory dual-probe monitoring. Am J Gastroenterol 1993; 88: 25-29

54 Wo JM, Hunter JG et al. Dual-channel ambulatory esophageal pH monitoring. A useful diagnostic tool? Dig Dis Sci 1997; 42: 2222-2226

55 Johnson LF, DeMeester TR. Twenty-four-hour pH monitoring of the distal esophagus. A quantitative measure of gastroesophageal reflux. Am J Gastroenterol 1974; 62: 325-332

${ }^{56}$ DeVault KR, Castell DO. A simplified technique for accurate placement of ambulatory pH probes. Am J Gastroenterol 1991; 86: 380 - 381

${ }^{57}$ Kahrilas PJ, Quigley EM. Clinical esophageal pH recording a technical review for pratice guideline development. Gastroenterology 1996; 110: $1982-1996$

${ }^{58}$ Dhiman RK, Saraswat VA, Mishra A et al. Inclusion of supine period in short-duration $\mathrm{pH}$ monitoring is essential in diagnosis of gastroesophageal reflux disease. Dig Dis Sci 1996; 41: 764-772

59 Dinelli M, Passaretti S, Di FI et al. Area under pH 4: a more sensitive parameter for the quantitative analysis of esophageal acid exposure in adults. Am J Gastroenterol 1999; 94: 3139-3144

60 Schindlbeck NE, Heinrich C, Konig A et al. Optimal thresholds, sensitivity and specificity of long-term pH-metry for the detection of gastroesophageal reflux disease. Gastroenterology 1987; 93: 85-90

${ }^{61}$ Schindlbeck NE, Ippisch H, Klauser AG et al. Which pH threshold is best in esophageal pH monitoring? Am J Gastroenterol 1991; 86: $1138-1141$ 
${ }^{62}$ Breumelhof R, Smout AJ. The symptom sensitivity index: a valuable additional parameter in 24-hour esophageal pH recording. Am J Gastroenterol 1991; 86: 160 - 164

63 Johnston BT, McDougall N, Collins JS et al. Association of symptoms with acid reflux during esophageal pH monitoring. Dig Dis Sci 1994; 39: $1593-1594$

${ }^{64}$ Weusten BL, Roelofs JM, Akkermans LM et al. The symptom-association probability: an improved method for symptom analysis of 24-hour esophageal pH data. Gastroenterology 1994; 107: $1741-1745$

${ }^{65}$ Wiener GJ, Richter JE, Copper JB et al. The symptom index: a clinically important parameter of ambulatory 24-hour esophageal pH monitoring. Am J Gastroenterol 1988; 83: 358 - 361

${ }^{66}$ Eckardt VF, Dilling B, Bernhard G. The impact of open access 24-h pHmetry on the diagnosis and management of esophageal reflux disease. Am J Gastroenterol 1999; 94: 616-621

${ }^{67}$ Kahrilas PJ, Clouse RE, Hogan WJ. American Gastroenterological Association. Technical Review on the clinical use of esophageal manometry. Gastroenterology 1994; 107: 1865 - 1884

${ }^{68}$ www.neurogastro.de

${ }^{69}$ Fang J, Bjorkman D. Prefundoplication testing: is manometry needed? Am J Gastroenterol 2002; 97 (4): 1056 - 1058 (no abstract available)

${ }^{70}$ Fibbe C, Layer P, Keller J et al. Esophageal motility in reflux disease before and after laparoscopic fundoplication. A prospective, randomized clinical and manometric study disease. Gastroenterology 2001; $121: 5-14$

${ }^{71}$ Gill RC, Bowes KL, Murphy PD et al. Esophageal motor abnormalities in gastroesophageal reflux and the effects of Fundoplication. Gastroenterology 1986; 91: $364-369$

${ }^{72}$ Gadenstatter M, Klingler A, Prommegger R et al. Laparoscopic partial posterior fundoplication provides excellent intermediate results in GERD patients with impaired esophageal peristalsis. Surgery 1999; 126: $548-552$

${ }^{73}$ Gadenstatter M, Klingler A, Klocker H et al. Long-term results of laparoscopic partial posterior fundoplication in patients with esophageal reflux and disorders of esophageal peristalsis. Wien Klin Wochenschr 2000; 112: $70-74$

${ }^{74}$ Lund RJ, Wetcher GJ, Raiser F et al. Laparoscopic Toupet Fundoplication for Gastroesophageal Reflux Disease With Poor Esophageal Body Motility. J Gastrointest Surg 1997; 1: 301 - 308

${ }^{75}$ Wetscher GJ, Glaser K, Gadenstaetter M et al. The effect of medical therapy and antireflux surgery on dysphagia in patients with gastroesophageal reflux disease without esophageal stricture. Am J Surg 1999; 177: 189-192

${ }^{76}$ Johnston BT, Troshinsky MB, Castell JA et al. Comparison of barium radiology with esophageal $\mathrm{pH}$ monitoring in the diagnosis of gastroesophageal reflux disease. Am J Gastroenterol 1996; 91: 1181 - 1185

77 Ott DJ, Glauser SJ, Ledbetter MS et al. Association of hiatal hernia and gastroesophageal reflux: correlation between presence and size of hiatal hernia and 24-hour $\mathrm{pH}$ monitoring of the esophagus. AJR Am J Roentgenol 1995; 165: 557-559

${ }^{78}$ Ferguson MK. Pitfalls and complications of antireflux surgery. Nissen and Collis-Nissen techniques. Chest Surg Clin N Am 1997; 7: 489-509

${ }^{79}$ Marshall RE, Anggiansah A, Manifold DK et al. Effect of omeprazole $20 \mathrm{mg}$ twice daily on duodenogastric and gastro-oesophageal bile reflux in Barrett's oesophagus. Gut 1998; 43: 603-606

${ }^{80}$ Vaezi MF, Richter JE. Synergism of acid and duodenogastroesophageal reflux in complicated Barrett's esophagus. Surgery 1995; 117: $699-704$
${ }^{81}$ Vaezi MF, Richter JE. Duodenogastro-oesophageal reflux. Baillieres Best Pract Res Clin Gastroenterol 2000; 14: 719-729

${ }^{82}$ Barrett MW, Myers JC, Watson DI et al. Detection of bile reflux: in vivo validation of the Bilitec fibreoptic system. Dis Esophagus 2000; 13: $44-50$

${ }^{83}$ Caldwell MT, Lawlor P, Byrne PJ et al. Ambulatory oesophageal bile reflux monitoring in Barrett's oesophagus. Br J Surg 1995; 82: $657-660$

${ }^{84}$ Champion G, Richter JE, Vaezi MF et al. Duodenogastroesophageal reflux: relationship to $\mathrm{pH}$ and importance in Barrett's esophagus. Gastroenterology 1994; 107: 747-754

${ }^{85}$ Kauer WK, Peters JH, DeMeester TR et al. Mixed reflux of gastric and duodenal juices is more harmful to the esophagus than gastric juice alone. The need for surgical therapy re-emphasized. Ann Surg 1995; 222: 525-531

${ }^{86}$ Vaezi MF, Lacamera RG, Richter JE. Validation studies of Bilitec 2000: an ambulatory duodenogastric reflux monitoring system. Am J Physiol 1994; 267: G1050-1057

${ }^{87}$ Cuomo R, Koek G, Sifrim D et al. Analysis of ambulatory duodenogastroesophageal reflux monitoring. Dig Dis Sci 2000; 45: 2463-2469

${ }^{88}$ Marshall RE, Anggiansah A, Owen WA et al. The relationship between acid and bile reflux and symptoms in gastro-oesophageal reflux disease. Gut 1997; 40: $182-187$

${ }^{89}$ Marshall RE, Anggiansah A, Owen WA et al. The temporal relationship between oesophageal bile reflux and $\mathrm{pH}$ in gastro-oesophageal reflux disease. Eur J Gastroenterol Hepatol 1998; 10: 385 - 392

${ }^{90}$ Menges M, Muller M, Zeitz M. Increased acid and bile reflux in Barrett's esophagus compared to reflux esophagitis, and effect of proton pump inhibitor therapy. Am J Gastroenterol 2001; 96: 331 - 337

${ }^{91}$ Nehra D, Howell P, Williams CP et al. Toxic bile acids in gastro-oesophageal reflux disease: influence of gastric acidity. Gut 1999; 44: $598-602$

92 Vaezi MF, Richter JE. Bile reflux in columnar-lined esophagus. Gastroenterol Clin North Am 1997; 26: 565 -582

${ }^{93}$ Dreuw B, Fass J, Buchin P et al. Combined pH measurement and multiple impedance variation assessments - validation of a new technique for detection of non-acid reflux in the esophagus. Langenbecks Arch Chir Suppl Kongressbd 1998; 115: 1143-1145

${ }^{94}$ Frieling T, Hermann S, Kuhlbusch R et al. Comparison between intraluminal multiple electric impedance measurement and manometry in the human oesophagus. Neurogastroenterol Motil 1996; 8: 45-50

${ }^{95}$ Nguyen HN, Silny J, Albers D et al. Dynamics of esophageal bolus transport in healthy subjects studied using multiple intraluminal impedancometry. Am J Physiol 1997; 273: G958 - 964

${ }^{96}$ Sifrim D, Holloway R, Silny J et al. Composition of the postprandial refluxate in patients with gastroesophageal reflux disease. Am J Gastroenterol 2001; 96: 647-655

${ }^{97}$ Sifrim D, Silny J, Holloway RH et al. Patterns of gas and liquid reflux during transient lower oesophageal sphincter relaxation: a study using intraluminal electrical impedance. Gut 1999; 44: 47-54

${ }^{98}$ Srinivasan R, Vela MF, Katz PO et al. Esophageal function testing using multichannel intraluminal impedance. Am J Physiol Gastrointest Liver Physiol 2001; 280: G457-462

${ }^{99}$ Katz PO. Review article: the role of non-acid reflux in gastro-oesophageal reflux disease. Aliment Pharmacol Ther 2000; 14: $1539-1551$

${ }^{100}$ Nguyen HN, Silny J, Matern S. Multiple intraluminal electrical impedancometry for recording of upper gastrointestinal motility: current results and further implications. Am J Gastroenterol 1999; 94: $306-317$

101 Tack J, Sifrim D. New techniques for the detection of gastro-oesophageal reflux. Dig Liver Dis 2000; 32 (Suppl 3): S245-248 